\title{
Lightness decrease and increase in square-wave grating
}

\author{
JIRO HAMADA \\ Tokushima University, Tokushima, Japan
}

\begin{abstract}
Lightness of low- and high-reflectance phases in square-wave gratings was measured and compared with that of uniform fields with the same reflectance. An enhancement of the lightness difference between the two phases of the grating was observed. The high-reflectance phase with $50 \%$ reflectance appeared to be darker than a uniform field of same reflectance, and even darker than a uniform field of $40 \%$ reflectance. As the difference in reflectance increased, however, the lightness at the high-reflectance phase changed from darker to lighter than that of the uniform field with the same reflectance. Thus, decrease and increase of lightness at the highreflectance phase of square-wave gratings depended upon the reflectance difference within the pattern. The enhancement of lightness difference and the lightness decrease of total area support the concept of a compound process of two types of inhibition: antagonistic excitation/ inhibition and nonantagonistic inhibition.
\end{abstract}

The relationship between area contrast and assimilation (von Bezold spreading effect) was studied quantitatively by Helson (1963). In that study, the observer judged the lightness of the two halves of a card ruled with white and black lines with instructions to compare the lightness of the intervening gray backgrounds. In terms of Helson's statement of contrast and assimilation, contrast occurred when the gray background between the white lines appeared darker than that between the black lines; conversely, assimilation occurred when the gray in the whitelined half was judged lighter than that in the blacklined half of the card. The results indicated that there was a continuum from assimilation to contrast with a transition zone between them. Assimilation could occur with stimuli having a very wide gray separation, that is, a 50 -mm gray separation between lines viewed at a distance of $3 \mathrm{~m}$, corresponding to a visual angle of $37 \mathrm{~min}$ of arc. The experimental procedure, however, clarified only the relative relationship between assimilation and contrast. It did not tell us whether the lightness of the gray backgrounds of the two halves of the card decreased or increased. We would like to know not only the relative relationship between the lightnesses, but also their absolute values.

This research was supported by a Grant in Aid for Scientific Research (No. 56710019) by the Japanese Ministry of Education. Prof. L. Spillmann and Dr. W. H. Ehrenstein of the Neurologische Universitäts-Klinik of Freiburg, i. Br., West Germany, provided many helpful comments and improved the readability of the manuscript. Prof. T. S. Aiba and Prof. S. Imai, of Hokkaido University, and Prof. M. Akita, of the Kyoto Institute of Technology, showed a continuing interest in this research. The author would like to express his grateful appreciation.
Up to this time, several investigators have adopted gratings to measure the modulation transfer function of the human visual system (Cornsweet, 1970). For example, Campbell and Robson (1968) and Patel (1966) measured contrast thresholds for detecting sine-wave gratings. Davidson (1968) developed a method of contrast matching. Although these techniques yielded many useful data, they cannot tell us the apparent lightness of gratings. Walker (1978) showed that the perceived brightness of the light phase of a square-wave grating was greater than that of a solid field of equal physical luminance; that is, brightness was enhanced at low spatial frequencies. At higher spatial frequencies, the perceived brightness of a grating was determined by its space-average luminances.

Hamada $(1978,1982,1983)$ found that when the simultaneous lightness contrast and the Craik-O'Brien illusion were induced in patterns with small reflectance differences, that is, less than about a $10 \%$ re-flectance difference, not only the low, but also the high-reflectance areas of the patterns became darker than those of the uniform fields with the same reflectances. Both the enhancement of lightness difference between the two areas and their lightness decrease may be explained by means of a compound process of antagonistic excitation/inhibition and nonantagonistic inhibition (Hamada, 1978, 1982, 1983). One type of inhibition arises from spatially antagonistic mechanisms, and the other arises from mechanisms that do not have antagonistic centersurround regions. Antagonistic inhibition corresponds to lateral inhibition of Békésy's (1968) neural units which depends upon the luminance and enhances the lightness difference. This kind of antagonistic inhibition may result as a psychophysical corre- 


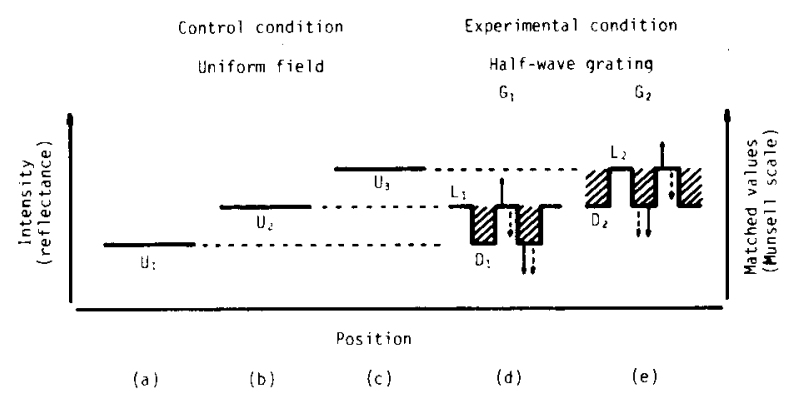

Figure 1. Illustration of uniform fields and half-wave gratings, and of hypothetical response changes by antagonistic and nonantagonistic processes. $U_{1}, U_{2}$, and $U_{3}$ are uniform flelds and $L_{1}$ and $L_{2}$ are light and $D_{1}$ and $D_{2}$ dark phases of the grating. Solid and dotted arrows indicate directions of hypothetical response changes caused by antagonistic excitation/inhibition and nonantagonistic inhibition, respectively.

late of the receptive fields, that is, as a perceptive field organization, as proposed by Jung and Spillmann (1970). Nonantagonistic inhibition, on the other hand, depends on the luminance difference and spreads widely over a large area to decrease the overall lightness of the pattern. The existence of nonantagonistic mechanisms has been suggested by Freund, Hennerici, and Rabenschlag (1977). They showed that a surround response changed to a center-type response when the center of the receptive field was darkened.

In the present study, pairs of half-wave rectified gratings, $G_{1}$ and $G_{2}$, are used (see Figure 1). One phase of the gratings is called the dark phase, $D_{1}$ or $\mathrm{D}_{2}$; the other is called the light phase, $\mathrm{L}_{1}$ or $\mathrm{L}_{2}$. The reflectance of $D_{1}$ and $L_{2}$ is the same as that of the corresponding uniform fields $U_{1}$ and $U_{3}$. The reflectance of $L_{1}$ and $D_{2}$ is the same as that of $U_{2}$. The reflectances of $D_{1}$ and $L_{2}$ are symmetrical about that of $U_{2}$. The logic of the compound processing model is illustrated by means of gratings and uniform fields, as depicted in Figure 1. Suppose that the reflectance of $U_{2}$ is locally decreased and forms the half-wave grating $G_{1}$, as indicated in Figure 1d. According to Békésy's neural units, the neural response at $L_{1}$ becomes greater than that of $U_{2}$, as indicated by the solid arrow. This refers to a lack of the inhibitory response from the hatched areas by the antagonistic process. On the other hand, the response at $D_{1}$ becomes weaker than that for $U_{1}$, as indicated by the solid arrow, because the antagonistic process induces an inhibitory response from $L_{1}$ to $D_{1}$. Thus, the enhancement of lightness difference is observed between $D_{1}$ and $L_{1}$. In addition to this process, a nonantagonistic process is assumed. This process reduces the neural response at the overall areas of the pattern, as indicated by the dotted arrows, since it does not have antagonistic center-surround regions.
Similarly, as shown in Figure 1e, when the halfwave grating $\left(\mathrm{G}_{2}\right)$ is formed by locally increasing the reflectance of $U_{2}$, the neural responses at $D_{2}$ and $L_{2}$ change, as compared with those for $\mathrm{U}_{2}$ and $\mathrm{U}_{3}$, in the direction indicated by solid arrows, because of the induction and reduction of antagonistic inhibition, respectively. Thus, the antagonistic excitation/inhibition should enhance the lightness difference between the two phases of $G_{2}$. Furthermore, nonantagonistic inhibition that depends on the reflectance difference spreads over the whole area of the grating and changes lightness so that the total area of the pattern becomes darker to a certain extent.

To sum up, in terms of the compound processing model, the lightness at the dark phase of the grating should be darker than that of a uniform field of the same reflectance due to antagonistic and nonantagonistic inhibition. On the other hand, the lightness change of the light phase depends upon the strength of two types of inhibition. Namely, when antagonistic inhibition is stronger than nonantagonistic inhibition, the lightness of the light phase of the grating should be greater than that of a corresponding uniform field. Here we perceived an increase in lightness. If, however, antagonistic inhibition is weaker than nonantagonistic inhibition, a decrease in lightness should occur at the light phase of the grating.

\section{METHOD}

\section{Apparatus}

Figure 2 shows the experimental apparatus. The front wall ( $40 \mathrm{~cm}$ wide and $40 \mathrm{~cm}$ high) of a viewing box was illuminated by $600 \mathrm{~lx}$ emitted by four quartz-iodine lamps $(6 \mathrm{~V}, 20 \mathrm{~W})$. On the wall was a test window (52 $\mathrm{mm}$ wide and $50 \mathrm{~mm}$ high, or $9.5 \times$ $9.2 \mathrm{deg}$ in visual angle). Behind the window, a drum rotated at high speed $\left(2,740 \mathrm{~min}^{-1}\right)$ to produce the test pattern. A Munsell
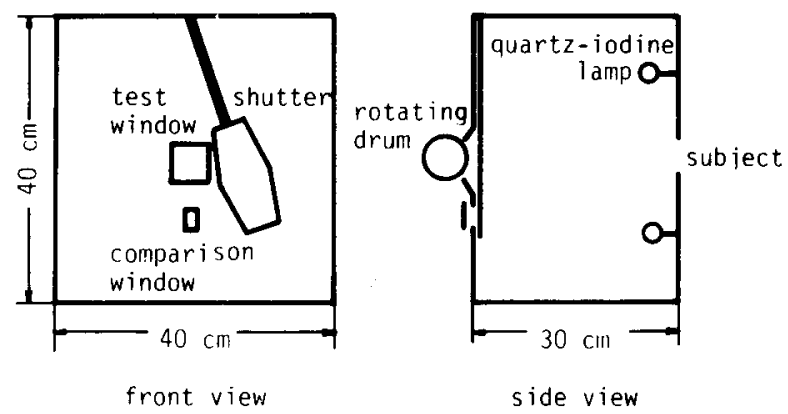

Figure 2. Illustration of the experimental apparatus. The front wall of the viewing box was illuminated by $600 \mathrm{Ix}$. On the wall was test window $(9.5 \times 9.2 \mathrm{deg}$ in visual angle). Behind the window, a drum rotated with high speed $(2,740$ min-1) to produce the teot pattern. A Munsell gray patch for compartson appeared in the comparison window (1.1 $\times 3.3 \mathrm{deg})$. The subject compared the Hightness of each Munsell gray patch with that of the test pattern. The pattern and the patch were simultaneously exposed by a shutter for 3 sec. 
gray patch for comparison appeared in the comparison window ( $6 \mathrm{~mm}$ wide and $18 \mathrm{~mm}$ high, or $1.1 \times 3.3 \mathrm{deg}$ ), which was located $42 \mathrm{~mm}$ below the test window. In order to determine the lightness of the test pattern, 33 Munsell gray patches were used. Values ranged from $1.5(2.0 \%$ reflectance) to $9.5(90 \%)$ in 0.25 steps. The distance between the subject and the front wall was $30 \mathrm{~cm}$. The test and the comparison windows were covered by a shutter which exposed simultaneously both the test and the comparison stimuli for $3 \mathrm{sec}$ on each trial. The front wall and the shutter were covered with black paper of 2.75 Munsell gray ( $5.5 \%$ reflectance).

\section{Pattern and Measurement of Its Reflectance}

Uniform fields were presented in the control condition, and half-wave gratings were presented in the experimental condition. They were produced by a fast rotating drum on which the stimulus patterns were pasted. Stimuli were printed on matte photographic paper $(60 \mathrm{~mm}$ wide and $190 \mathrm{~mm}$ long) and had a reflectance of $2.3 \%$ for black and $78 \%$ for white. The luminance and the reflectance values for the black and white parts were measured at the observer's position using a MacBeth illuminometer. The mean reflectance calculated according to Talbot's law was used as an index of stimulus intensity. In Experiments 1 and 2, there were four widths for the cycle of grating, 8, 24, 72, and $110 \mathrm{~min}$, corresponding to spatial frequencies of $7.5,2.5,0.83$, and $0.55 \mathrm{cycles} / \mathrm{deg}$, respectively. In both experiments, the reflectance of one phase $\left(\mathrm{L}_{1}\right.$ or $\mathrm{D}_{2}$ ) of the half-wave grating and uniform field $\left(\mathrm{U}_{2}\right)$ were fixed at $40 \%$. All gratings were oriented vertically.

Experiment 1. Three uniform fields with reflectances of $30 \%$ $\left(\mathrm{U}_{1}\right), 40 \%\left(\mathrm{U}_{2}\right)$, and $50 \%\left(\mathrm{U}_{3}\right)$ served as controls. The reflectances of the dark phase $\left(D_{1}\right)$ and the light phase $\left(L_{2}\right)$ were $40 \%-10 \%$ and $40 \%+10 \%$, respectively. We refer to a pair of half-wave gratings as the $40 \% \pm 10 \%$ reflectance condition. The subject matched the lightnesses of the uniform fields as well as of the dark phases $\left(D_{1}\right.$ and $\left.D_{2}\right)$ and the light phases $\left(L_{1}\right.$ and $\left.L_{2}\right)$ of the gratings.

Experiment 2. A uniform field with a reflectance of $40 \%\left(\mathrm{U}_{2}\right)$ served as a control. The reflectances of the dark phases $\left(D_{1}\right)$ were $40 \%-12 \%, 40 \%-24 \%$, and $40 \%-36 \%$, and those of the light ones $\left(L_{2}\right) 40 \%+12 \%, 40 \%+24 \%$, and $40 \%+36 \%$. Subjects matched the lightnesses of the uniform field and the light phase $\left(L_{1}\right)$ and the dark phase $\left(D_{2}\right)$ with a reflectance of $40 \%$.

\section{Procedure}

Six males, students in an undergraduate course, participated in the experiments, three in Experiment 1 and three in Experiment 2. All had normal or corrected-to-normal vision. The subjects used a chinrest and observed both the test and the comparison fields binocularly. The subjects compared the lightness of each Munsell gray patch with that of the test pattern. They were advised to shift fixation between the test and the comparison fields every $3 \mathrm{sec}$. The method of limits was used. Test patterns were presented in a random sequence. The sequences for the dark and the light phases and for the descending and the ascending series were counterbalanced. Following practice trials, each subject participated in six experimental sessions.

\section{RESULTS}

Despite their individual variability, ${ }^{1}$ the data of both experiments are consistent with respect to their general trend. The average data obtained under the $40 \% \pm 10 \%$ reflectance condition are shown in Figures $3 a$ and $3 b$ as a function of cycle width of the grating. Data obtained under the other three reflectance conditions are shown in Figures $4 a, 4 b$, and $4 c$. Vertical bars indicate $\pm 1 \mathrm{SD}$.

\section{Experiment 1}

Lightness of the uniform field. The reflectances of the uniform fields were $30 \%, 40 \%$, and $50 \%$. According to the notation of the Munsell color system, these values should have corresponded to Munsell values of 6.0, 6.8, and 7.5. However, they were matched with Munsell values of 5.6, 6.2, and 6.7, as indicated by the dotted, the solid, and the broken lines in Figure 3, respectively. This discrepancy might be caused by the difference in total area between the uniform field and the comparison field. It does not matter, however, since only relative values are considered in the present study. According to Talbot's law as applied in the spatial domain by Walker (1978), if we compare the lightness of a uniform field with the space-average reflectance of the grating, $G_{1}$ or $G_{2}$, the matched Munsell value for the field should fall between the data for $U_{1}$ and $U_{2}$ or $U_{2}$ and $U_{3}$, as indicated by the small filled circles in Figure 3. We call this the calculated Munsell value.

Lightness decrease of total area and paradoxical lightness decrease of light phase. As shown in Figure 3a, the matched Munsell values were smaller for both the light phase, $L_{1}$ (filled circle, $\bullet$ ), and the dark phase, $D_{2}$ (open circle, $O$ ), than the matched one for the uniform field (solid line) with the same $40 \%$ reflectance $(p<.01)$ and the calculated one

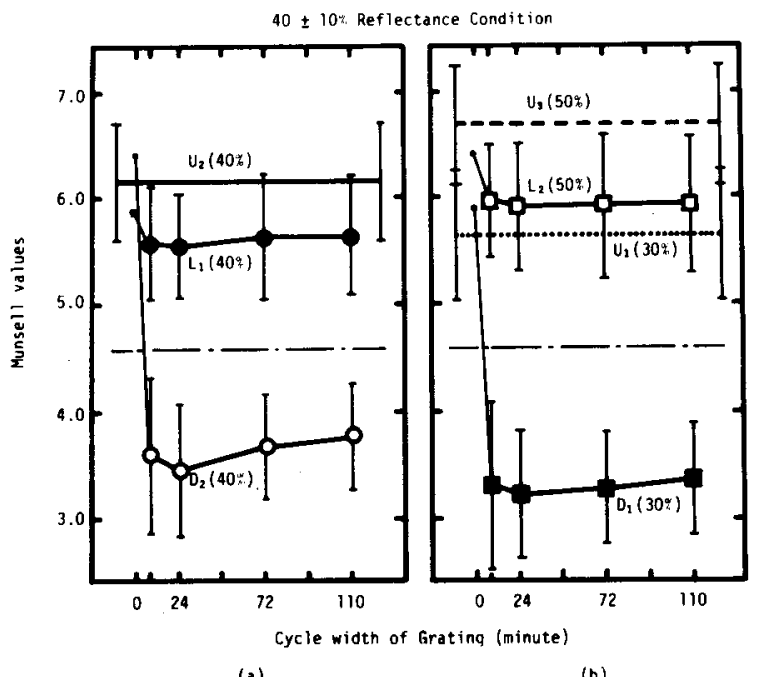

Figure 3. Average Munsell values as a function of cycle width of the grating. Lightnesses of the light phase $\left(L_{1}\right.$; fllled circle, $\bullet$ ) and the dark phase $\left(D_{2}\right.$; open circle, $\left.O\right)$ of pairs of half-wave grating $\left(G_{1}\right.$ and $G_{2}$ ) and uniform field $\left(U_{2}\right.$; solid line) are shown in (a); those of the dark phase ( $D_{1}$; filled square, $\left.D\right)$, the light phase $\left(\mathrm{L}_{2}\right.$; open square, $\square$ ), and uniform fields ( $U_{1}$ and $U_{3}$; dotted and broken lines) are shown in (b). Small filled circles indicate calcuInted Munsell values for uniform field with space-average reflectance of a half-wave grating. 


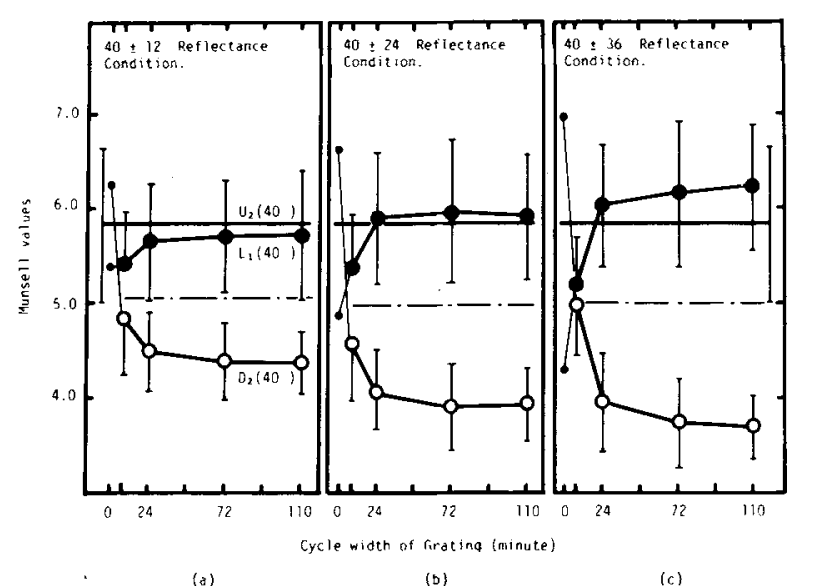

(a)

(b)

(c)

Figure 4. Average Munsell values as a function of cycle width of the grating. Lightnesses of the light phase $\left(L_{1} ;\right.$ filled circle, $\left.\bullet\right)$ of a half-wave grating $\left(G_{1}\right)$, the dark phase $\left(D_{2}\right.$; open circle, $\left.O\right)$ of a half-wave grating $\left(G_{2}\right)$, and a uniform field $\left(U_{2}\right.$; solid line) are measured. Small fllled circles indicate calculated Munsell values for a uniform field with space-average reflectance of a half-wave grating.

(small filled circle) for the space-average reflectance field. Similarly, as shown in Figure 3b, the matched Munsell values for the dark phase, $D_{1}$ (filled square, $\square$ ), and for the light phase, $\mathrm{L}_{2}$ (open square, $\square$ ), were smaller $(p<.01)$ than the matched values for the corresponding uniform fields (dotted and broken lines). These results imply that the lightness of both phases of the grating, $G_{1}$ or $G_{2}$, is lower than that of the corresponding uniform fields. Furthermore, the lightness of the light phase, $\mathrm{L}_{2}$ (Figure $3 \mathrm{~b}$, open squares), of $40 \%+10 \%$ reflectance was lower ( $p<.01$, or $p<.05$ for the cycle width of $8 \mathrm{~min}$ ) than that of the uniform field (Figure 3a, solid line) of $40 \%$ reflectance. A similar phenomenon was reported by Hamada (1983). We would like to call this effect a paradoxical lightness decrease, because increasing the reflectance of the uniform field locally to form the light phase resulted in a lightness decrease in spite of the reflectance increment.

Enhancement of lightness difference. The difference in matched Munsell values between uniform fields of $30 \%$ and $40 \%$ reflectance, on the one hand, and between $40 \%$ and $50 \%$ reflectance, on the other, was about 0.5 Munsell unit, as indicated in Figure 3. In contrast, the difference in matched Munsell values between the light and the dark phases (filled circle and filled square for $G_{1}$, open circle and open square for $\mathrm{G}_{2}$ ) was about 2.5. These results imply that the lightness differences between the light and dark phases of the grating were enhanced in comparison with those found for uniform fields of equal reflectances.

Symmetry in lightnesses between light and dark phases. The data for each kind of phase were pooled, and their mean was calculated. The means for $D_{1}, L_{1}$, $D_{2}$, and $L_{2}$ were $3.3,5.6,3.6$, and 5.9 , respectively. If we sort them into two sets, $L_{1}$ and $D_{2}$, with $40 \%$ reflectance, and $D_{1}(40 \%-10 \%)$ and $L_{2}(40 \%+10 \%)$, with a symmetrical relationship in reflectance, the mean of means for the former as well as for the latter is 4.6 (see center lines in Figures $3 a$ and $3 b$ ). This implies that the matched Munsell values for $L_{1}$ and $D_{2}$ with $40 \%$ reflectance and those for $D_{1}(40 \%-$ $10 \%)$ and $L_{2}(40 \%+10 \%)$ show an approximately symmetrical relationship in Munsell units divided by a center line of 4.6. Similarly, the matched Munsell values for $U_{1}$ and $U_{3}$ show the same symmetrical relationship by a center line of 6.2 , which is the matched value for $U_{2}$.

\section{Experiment 2}

Lightness of uniform field. The matched value of $5.8^{2}$ for the uniform field was again smaller than the value of the corresponding physical reflectance. The decrease was 1.0 in Munsell value. We tentatively assumed that the discrepancy of a 1.0 decrease should appear in the data for uniform fields of any reflectance. Then we calculated the Munsell values for the six uniform fields whose reflectance equaled the space-average reflectance of the half-wave gratings, $G_{1}$ and $G_{2}$, and obtained the calculated Munsell values indicated by the small filled circles in Figure 4.

Lightness decrease and increase of light phases. For all light phases, $L_{1}$ (filled circle, $\bullet$ ) of $G_{1}$, the matched values were smaller at a cycle width of $8 \mathrm{~min}$ than they were for the uniform field $(p<.01)$. However, at and above a cycle width of $24 \mathrm{~min}$, the lightness of the light phases systematically increased above that of the uniform fields. For the $40 \%$ $12 \%, 40 \%-24 \%$, and $40 \%-36 \%$ reflectance conditions, the lightness at the light phases was first below, then nearly equal to, and finally above $(p<.01)$ that of the uniform field.

Relationship between lightness of light and dark phases. In order to investigate the relationship between lightness at $\mathrm{L}_{1}$ and $\mathrm{D}_{2}{ }^{3}$ with $40 \%$ reflectance, the calculated Munsell value was connected with the matched one by thin lines, as shown in Figure 4. All pairs of the thin lines cross below the cycle width of $8 \mathrm{~min}$. The lightness at the dark phase $\mathrm{D}_{2}$ of the grating $\left(G_{2}\right)$ was darker than the light phase $L_{1}$ of the grating $\left(G_{1}\right)$ above the crossing point. The greater the reflectance difference between the light and the dark phases in each grating, the greater is the amount of reversal of lightness.

In the same way as in Experiment 1, the matched Munsell values for the dark and the light phases $(\mathbf{4 0 \%})$ show a symmetrical relationship. As shown in Figure 4, the center lines for three sets of curves are similar and about 5.0 in Munsell value. 


\section{DISCUSSION}

The data plotted in Figure 4 indicated that the matched Munsell values for the dark and light phases with $40 \%$ reflectance initially fell or rose, and tended to level off at about the cycle width of $24 \mathrm{~min}$, corresponding to a bar width of $12 \mathrm{~min}$. Jung and Spillmann (1970) and Spillmann $(1971,1981)$ proposed the term "perceptive fields" to denote the psychophysical correlate of center-surround antagonism within receptive fields of the human retina. Spillmann (1971) reports $17.8 \mathrm{~min}$ of arc for the total field diameter and $4 \mathrm{~min}$ for the center by means of contrast illusion in Hermann grid. Ransom-Hogg and Spillmann (1980) report less than 9 min for the center and $1 \mathrm{deg}$ for the total field using the Westheimer paradigm. The center size corresponds closely to the bar width of $12 \mathrm{~min}$, where the matched Munsell values level off. This correspondence may be explained as follows. With the enlargement of the phase width, area summation occurs within the center of the perceptive field. Then the lightnesses of the dark and the light phases, $D_{1}$ and $L_{2}$, become darker and lighter up to the phase width at the value of $12 \mathrm{~min}$, corresponding to the center size of perceptive fields.

The lightness increase for the $40 \%-36 \%$ reflectance condition is consistent with Walker's results (1978), and it can be qualitatively explained by the antagonistic mechanism. On the other hand, the paradoxical lightness decrease under the $40 \%+10 \%$ reflectance condition as well as the lightness decrease observed for the light phases of the $40 \%-10 \%$ and $40 \%-12 \%$ reflectance conditions have not been reported before and cannot be explained by the antagonistic one. Here it seems to be necessary to refer to nonantagonistic inhibition, a concept that has been introduced by Hamada $(1978,1982)$. The following assumptions are made: When the reflectance difference within the pattern is small, the antagonistic inhibition is weaker than the nonantagonistic one. When it is large, the former is stronger than the latter. The lightness decrease or increase observed at the light phase of gratings depends on the amount of reflectance difference. Thus, both the enhancement of lightness difference between the two phases of halfwave gratings and the lightness decrease or increase at the light phase are qualitatively predicted by the compound process of antagonistic and nonantagonistic mechanisms.

There appears to be a symmetrical relationship between lightness at the dark and light phases of any pair of half-wave gratings. The center line is lower than the matched Munsell value for the uniform field of $40 \%$ reflectance, as shown in Figures 3 and 4, respectively. One may assume that nonantagonistic inhibition decreases the center line for a pair of curves for half-wave gratings, and the decrease is constant for any reflectance difference condition. Then the compound processing model predicts the lightness change at the light phase $L_{1}$ of the gratings $\left(G_{1}\right)$ as follows. If the reflectance difference within the half-wave grating is less than $10 \%$, the lightness at $L_{1}$ falls from the calculated Munsell value for the space-average reflectance field in a very similar fashion for all cycle widths (see Figure 3a). On the other hand, when the difference is about $12 \%, 24 \%$, and $36 \%$, the lightness rises as a function of cycle width (see Figures 4a-4c).

The comparison between the matched and the calculated Munsell values for $L_{1}$ and $D_{2}$ reveals that the reversal of lightness at the phases occurs below a cycle width of $8 \mathrm{~min}$. The reversal of lightness may correspond to the transition from assimilation to contrast described by Helson (1963). The lightness decrease or increase and the reversal of lightness obtained in our results may suggest that the lightness of the total area of the pattern may change jointly with assimilation or contrast. In terms of our model, both assimilation and contrast result from antagonistic excitation/inhibition and nonantagonistic inhibition.

\section{CONCLUSION}

The results, that is, the lightness decrease and the lightness increase within a half-wave grating, support the concept of a compound process of antagonistic excitation/inhibition and nonantagonistic inhibition (Hamada, 1978, 1982). The antagonistic mechanism induces the enhancement of lightness difference of the grating phases, whereas the nonantagonistic process decreases the lightness of total area.

\section{REFERENCES}

BÉKésy, G. v. (1968). Mach- and Hering-type lateral inhibition in vision. Vision Research, 8, 1483-1499.

Campbell, F. W., \& Robson, J. G. (1968). Application of Fourier analysis to the visibility of gratings. Journal of Physiology, London, 197, 551-566.

Cornsweet, T. N. (1970). Visual perception. New York: Academic Press.

Davidson, M. (1968). Perturbation approach to spatial brightness interaction in human vision. Journal of the Optical Society of America, 58, 1300-1308.

Freund, H.-J., Hennerici, M., \& Rabenschlag, U. (1977). Reversal of surround- into centre-type responses of cat retinal ganglion cells by local darkening of the receptive field centre. Vision Research, 17, 487-494.

HAmADA, J. (1978). Antagonistic and non-antagonistic processes for the lightness perception. Hokkaido Behavioral Science Report, Series $P$, No. 4, Sapporo.

Hamada, J. (1982). The contour enhancement effects produced by darkening effects. In H.-G. Geissler \& P. Petzold (Eds.), Psychophysical judgment and the process of perception (pp. 132-139). Amsterdam: North-Holland.

HAMADA, J. (1983). Lightness decrease of total area accompany- 
ing simultaneous lightness contrast. Japanese Journal of Psychology, 54, 2, 115-122.

Helson, H. (1963). Studies of anomalous contrast and assimilation. Journal of the Optical Society of America, 53, 179-184.

Jung, R., \& Spillman, L. (1970). Receptive-field estimation and perceptual integration in human vision. In F. A. Young \& D. B. Lindsley (Eds.), Early experience and visual information processing in perceptual and reading disorders (pp. 181-197). Washington, DC: National Academy of Sciences.

Patel, A. S. (1966). Spatial resolution by the human visual system. The effect of mean retinal illumination. Journal of the Optical Society of America, 56, 689-694.

Ransom-Hoga, A., \& Spillmann, L. (1980). Perceptive field size in fovea and periphery of the light- and dark-adapted retina. Vision Research, 20, 221-228.

Spillmann, L. (1971). Foveal perceptive fields in the human visual system measured with simultaneous contrast in grids and bars. Pflügers Archiv für die Gesamte Physiologie, 326, 281-299.

SpillmanN, L. (1981). Illusion of contrast, brightness, color and motion and their neurophysiological interpretation. Freiburger Universitätsblätter, 74, 73-77.

WALkER, J. T. (1978). Brightness enhancement and Talbot level in stationary grating. Perception \& Psychophysics, 23, 356-359.

\section{NOTES}

1. In the present study, we used a two-factor analysis of variance among three subjects and between two conditions. In every case there were highly significant differences $(p<.01)$ among subjects.

2. The matched Munsell value for uniform field $\left(U_{1}\right)$ with $40 \%$ reflectance in Experiment 2 was smaller than that in Experiment 1. This discrepancy might result from the difference between subjects participating in the two experiments.

3. The matched Munsell values for the dark phase $\left(D_{2}\right)$ of grating $\left(\mathrm{G}_{2}\right)$ under the $40 \%+12 \%$ reflectance condition in Experiment 2 were considerably greater than those under the $40 \%+$ $10 \%$ reflectance condition in Experiment 1 . This discrepancy might result from the difference between subjects participating in the two experiments and the difference in the subjects' task; that is, in Experiment 1 the subjects successively judged the lightnesses of both phases of grating, but in Experiment 2 they judged only one phase of it.

(Manuscript received June 3, 1983; revision accepted for publication November 21, 1983.) 